\title{
EDITORIAL AND COMMENT \\ Unconscious Bias and Real-World Hypertension Outcomes: Advancing Disparities Research
}

\author{
Joseph Ravenell, MD, MS and Gbenga Ogedegbe, MD, MPH, MS \\ Center for Healthful Behavior Change, Division of Health and Behavior, Department of Population Health, New York University School of \\ Medicine, New York, NY, USA.
}

J Gen Intern Med 29(7):973-5

DOI: $10.1007 / \mathrm{s} 11606-014-2849-2$

(c) Society of General Internal Medicine 2014

I n 2003, in its landmark report Unequal Treatment, the Institute of Medicine (IOM), after examining the evidence and theories up to that time, boldly proclaimed: "Indirect evidence indicates that bias, stereotyping, prejudice, and clinical uncertainty on the part of healthcare providers may be contributory factors to racial and ethnic disparities in healthcare. Prejudice may stem from conscious bias, while stereotyping and biases may be conscious or unconscious, even among the well intentioned (emphasis added).",

Since that report, in which the IOM called for more research "to identify how and when these processes [bias, stereotyping] occur", several studies have examined the role of implicit or unconscious bias in physician behavior and decision-making, and made important contributions to advancing our understanding of how bias may contribute to health disparities. Many of these studies were conducted by measuring physicians' level of implicit bias against racial/ethnic minorities using the well-validated Implicit Associations Test $(I A T),{ }^{2}$ and then linking the level of bias to provider behavior or decision making in clinical simulations, scenarios and vignettes. ${ }^{3}$ However, when observing how a physician behaves or responds to a hypothetical vignette or scenario, it is not physician behavior that is being measured; rather, it is the physician's behavioral intention in the given scenario or vignette, which is not always predictive of actual behavior. Thus, more research that examines physicians' unconscious bias in the context of their actual behavior (with actual patients) is needed.

In this issue of JGIM, Blair et al. took an important step forward in addressing this gap. ${ }^{3}$ In a well-designed study, the authors administered IATs to 138 primary care physicians across two health systems in Colorado. Next, via electronic medical record review, hypertension-related process and clinical outcomes of 4,794 Black, Latino and white patients cared for by the 138 participating physicians were examined and modeled for associations with physician biases. With a

Published online April 8, 2014 focus on well-established measures such as treatment intensification, medication adherence and blood pressure (BP) control, Blair et al. moved the research on implicit bias out of the hypothetical realm and into the real world.

Notably, $70 \%$ of the physicians showed some implicit bias against Blacks or Latinos, and more than $40 \%$ of them reported moderate-to-strong levels of bias against Blacks and Latinos. Treatment intensification did not significantly differ across Black, Latino and white patients; Blacks and Latinos had lower medication adherence compared to whites, and Blacks had worse BP control than whites and Latinos. None of the outcomes was associated with the noted implicit bias.

Although the authors did not find the hypothesized relationship between physician unconscious bias and the hypertension-related outcomes, in our view, this paper is still noteworthy and influential, for the following reasons. First, among the participating physicians, the rate of implicit bias against Blacks or Latinos was quite high. This exactly mirrors a study of over 1 million Americans, in which $70 \%$ were found on the IAT to have implicit bias against racial/ethnic minorities. ${ }^{4}$ The similarity in the prevalence of implicit bias between physicians and the general population is congruent with other studies that have found that physicians hold stereotypes and biases, often unconsciously, based on race, gender and other characteristics. ${ }^{5}$ This is not surprising, because like the general population, physicians are human, and are subject to the same socializing influences, ${ }^{1}$ such as constant negative portrayals of members of racial/ethnic minorities by popular and social media. Second, in spite of the high prevalence of implicit bias among the physicians, the authors found no association between clinician bias and hypertension outcomes in minority patients. This finding is important because it suggests, as the authors note, "Primary care has a number of features that are likely to mitigate the impact of bias." These features include the opportunity for strong working relationships between patients and physicians; opportunities afforded by the longitudinal nature of primary care to address the patients' medical needs in ways that avoid the influence of bias; strong expectations for meeting hypertension control guidelines at the organiza- 
tional level; and lastly, checks and balances that occur in primary care teams (e.g., physicians, nurses and pharmacists), and in integrated health care systems. ${ }^{3}$ Such structural 'safeguards' may be important for minimizing the potential negative impact of physician bias.

There are some factors that may help to explain the lack of association between implicit bias and hypertension-related outcomes in this study. First, the measures that were included in the study, while they are among the commonly used measures in their respective categories, are not perfect. The IAT, one of the gold standard instruments for measuring implicit social cognition, has been criticized for not actually measuring implicit racial bias, but rather measuring in-group versus out-group preference. ${ }^{6}$ Also, after personally taking the IAT ourselves, it is not clear to us whether the IAT is measuring preference for whites, or negative bias against blacks. This distinction is important, because preference for one group is not the same as a negative bias against another group. Second, as noted by the authors, pharmacy refill data is an imperfect proxy for medication adherence. Though we understand the rationale for using this easily accessible measure in this pragmatic study that used electronic medical record queries, this proxy for patient-level medication adherence may not be accurate or sensitive enough to capture the impact of a complex physician-level characteristic like implicit bias. Lastly, other factors that were not considered in the current study that may mitigate or augment the effect of provider bias on outcomes include race concordance or discordance in doctor-patient dyads, patient trust in physicians, patients' perceptions of historic and current racial discrimination both in the medical setting and the larger society, and racism-related coping. Patients with certain coping styles may be able to overcome the expression of a provider's bias or other adversity and achieve favorable outcomes in spite of his or her provider.

Additional questions to consider for advancing and interpreting this kind of research include: what is the optimal observation period, and how much interaction must a patient have with the same provider in order to be affected by implicit bias? Blair et al. examined outcomes during a 3year observation period, and included patients with at least three visits over the observation period. Though one visit per year hardly seems like enough of a 'dose' of a provider to have an impact on outcomes like BP control, prior studies have reported one instance of discrimination or disrespect (which are often the expression of bias) in the medical setting can be associated with adverse consequences for the patient. Blanchard and Lurie found that black patients who reported experiences of disrespect in the medical setting were significantly less likely to follow their doctor's advice. $^{7}$ The King County Ethnicity and Health
Survey found that among patients who perceived racial discrimination while seeking healthcare, many were more hesitant to seek healthcare services. ${ }^{8}$

One note of caution; the absence of a statistical relationship between clinician bias and the hypertension outcomes in this study, even with its noted limitations, should in no way detract from the importance of this area of investigation, for two reasons. First, the adverse impact of implicit bias and its expression has been demonstrated in previous studies. In a recent study, Blair et al. found that black patients reported less patient-centered treatment from physicians with higher levels of bias compared to providers with lower levels of bias on the IAT. ${ }^{9}$ Thus, implicit bias can adversely affect the doctor-patient relationship, especially among minority patients. Second, it is possible that even if implicit biases cannot easily be changed, the manner in which implicit bias is expressed (i.e., discrimination or differential treatment) can be altered with situational goals, motivation and behavioral strategies. ${ }^{10}$ Burgess et al. (in a previous issue of the journal), highlighted evidence from social cognitive psychology that unconscious bias can be counteracted with increased awareness, sufficient motivation and cognitive resources. ${ }^{11}$ Thus, interventions to reduce the expression of bias may subsequently improve physicians' relationships with minority patients in general, and should continue to be a research priority.

The current paper by Blair et al. provides a model for linking measures of provider implicit bias to actual clinical practice with accepted metrics (i.e., hypertension treatment and control), that can be understood by physicians, payors and policymakers. Future replication of this study [in clinical populations that differ from the ones in the current study] for hypertension and other chronic disease outcomes will contribute to a better understanding of provider-level factors as mechanisms of racial disparities in health outcomes and burden.

Acknowledgements: Dr. Ogedegbe's work was partially funded by a K24 award from NHLBI: K24HL111315.

Corresponding Author: Joseph Ravenell, MD, MS; Center for Healthful Behavior Change, Division of Health and Behavior, Department of Population Health, New York University School of Medicine, 227 East 30th Street, 6th Floor, New York, NY 10016, USA (e-mail: joseph.ravenell@nyumc.org).

\section{REFERENCES}

1. Smedley BD, Stith AY, Nelson AR, eds. Unequal treatment: confronting racial and ethnic disparities in health care. Washington, D.C.: National Academies Press; 2003.

2. Greenwald AG, McGhee DE, Schwartz JL. Measuring individual differences in implicit cognition: the implicit association test. J Personal Soc Psychol. 1998;74:1464-1480.

3. Blair IV, Steiner JF, Hanratty R, et al. An Investigation of Associations Between Physicians' Ethnic or Racial Bias and Hypertension Treatment, Medication Adherence and Blood Pressure Control. J Gen Intern Med. DOI: $10.1007 / \mathrm{s} 115606-014-2795-Z$ 
4. Nosek BA, Smyth FL, Hansen JJ, et al. Pervasiveness and correlates of implicit attitudes and stereotypes. Eur Rev Soc Psychol. 2007; 18:36-88.

5. van Ryn M. Research on the provider contribution to race/ethnicity disparities in medical care. Med Care. 2002;40:I140-I151.

6. Kaufman SB. Does the Implicit Association Test (IAT) Really Measure Racial Prejudice? Probably Not. Psychol Today. 2011.

7. Blanchard J, Lurie N. R-E-S-P-E-C-T: patient reports of disrespect in the health care setting and its impact on care. J Fam Pract. 2004;53:721-730
8. Hobson w. Racial discrimination in Health Care Interview Project: Special Report. 2001.

9. Blair IV, Steiner JF, Fairclough DL, et al. Physicians' implicit ethnic/ racial bias and perceptions of care among black and latino patients. Ann Fam Med. 2013;11:43-52.

10. Lai CK, Hoffman KM, Nosek BA. Reducing implicit prejudice. Soc Personal Psychol Compass. 2013;7:315-330.

11. Burgess D, van Ryn M, Dovidio J, Saha S. Reducing racial bias among health care providers: lessons from social-cognitive psychology. J Gen Intern Med. 2007;22:882-887. 